\title{
Fragmented hyaluronan is an autocrine chemokinetic motility factor supported by the HAS2-HYAL2/ CD44 system on the plasma membrane
}

\author{
TERUYOSHI SAITO ${ }^{1}$, HIDETADA KAWANA ${ }^{2}$, KAZUHIKO AZUMA ${ }^{1}$, AKIHIRO TOYODA ${ }^{1}$, \\ HIROSHI FUJITA $^{3}$, MOTOO KITAGAWA ${ }^{1}$ and KENICHI HARIGAYA ${ }^{1}$
}

\author{
${ }^{1}$ Department of Molecular and Tumor Pathology, Graduate School of Medicine, Chiba University, 1-8-1 Inohana, \\ Chuo-ku, Chiba 260-8670; ${ }^{2}$ Division of Surgical Pathology, Chiba Cancer Center Research Institute, \\ 666-2 Nitona-Cho, Chuo-ku, Chiba 260-8717; ${ }^{3}$ Glycoconjugate Research Center, Kurihama Plant, \\ Seikagaku Corporation, 7-3-1 Kurihama, Yokosuka-shi, Kanagawa 239-0831, Japan
}

Received May 3, 2011; Accepted June 11, 2011

DOI: 10.3892/ijo.2011.1114

\begin{abstract}
Hyaluronan (HA) is synthesized by HA synthase (HAS) 1, HAS2 and HAS3, and degraded by hyaluronidase (HYAL) 1 and HYAL2 in a CD44-dependent manner. HA and HYALs are intricately involved in tumor growth and metastasis. Random cell movement is generally described as chemokinesis, and represents an important step at the beginning of tumor cell liberation from the primary site. To investigate the roles of HAS2 and HYAL2/CD44 in cell motility, we examined HeLa-S3 cells showing spontaneous chemokinesis. HeLa-S3 cells expressed HAS2 and HAS3. siRNA-mediated knockdown of HAS2 decreased spontaneous chemokinesis of HeLa-S3 cells. Although HeLa-S3 cells secreted $50 \mathrm{ng} / \mathrm{ml}$ of high molecular weight (HMW)-HA (peak: $990 \mathrm{kDa}$ ) into the culture supernatant after $6 \mathrm{~h}$ of culture, exogenously added HMW-HA did not enhance spontaneous chemokinesis of the cells. These observations suggested that HeLa-S3 cells may have a self-degrading system for HA to regulate their spontaneous chemokinesis. To examine this possibility, we investigated the effects of siRNA-mediated knockdown of HYAL2 or CD44 on the spontaneous chemokinesis of HeLa-S3 cells. Knockdown of either molecule decreased the spontaneous chemokinesis of the cells. Low molecular weight (LMW)-HA $(23 \mathrm{kDa})$ reversed the HYAL2 siRNA-mediated reduction in spontaneous chemokinesis of HeLa-S3 cells to the level in control cells stimulated with the same HA. These findings indicate that the HAS2-HYAL2/CD44 system may support spontaneous chemokinesis of human cancer cells through
\end{abstract}

Correspondence to: Dr Kenichi Harigaya, Department of Molecular and Tumor Pathology, Graduate School of Medicine, Chiba University, 1-8-1 Inohana, Chuo-ku, Chiba 260-8670, Japan

E-mail: harigaya@faculty.chiba-u.jp

Key words: hyaluronan, hyaluronan synthase 2, hyaluronidase 2, CD44, chemokinesis self-degradation of HMW-HA to produce LMW-HA by an autocrine mechanism. Consequently, our study may further expand our understanding of HA functions in cancer.

\section{Introduction}

Hyaluronan (HA) is a non-sulfated linear glycosaminoglycan that consists of repeating disaccharide subunits of glucuronic acid and $\mathrm{N}$-acetylglucosamine. It has a ubiquitous distribution and is the major type of glycosaminoglycan present in the extracellular matrix (1). High molecular weight (HMW)-HA exists in normal tissues and contributes to local homeostasis by suppressing cell proliferation, migration, angiogenesis, inflammation and immunologic reactions (2-4). On the other hand, low molecular weight (LMW)-HA is associated with inflammation and tumor progression $(5,6)$. In fact, HA interacts with several cell-surface receptors, such as CD44 and RHAMM (7), and HA-CD44 interactions can lead to the activation of intracellular signaling pathways that affect the proliferation, migration and invasion of cancer cells (8-11).

Recent reports have made it clear that the balance of HA synthesis and degradation is critical for tumor progression $(12,13)$. HA is synthesized on the cytoplasmic surface of the plasma membrane by hyaluronan synthase (HAS) 1, HAS2 and HAS3, and extruded into the extracellular space $(14,15)$. The catalytic activities of the three HAS enzymes are different. HAS1 and HAS2 produce HA of 200-2000 kDa, whereas HAS3 mainly synthesizes HA of 100-1000 kDa (14). Knockdown of the HAS genes in tumor cells was reported to inhibit their proliferation, invasion and motility in vitro and tumor growth and metastasis in vivo (16-22). In particular, it has been reported that the levels of HAS2 expression are often correlated with malignant behavior in various cancer cells such as breast cancer, fibrosarcoma and osteosarcoma cells (17,23-25). Furthermore, HAS2 is a key molecule for the induction of the epithelial-mesenchymal transition (EMT) with HA assembly (26-30).

In vivo, HMW-HA is degraded to LMW-HA via oxygen radicals and enzymatic degradation by hyaluronidases (HYALs). 
Six HYAL-like genes have been identified in humans. Specifically, HYAL1,HYAL2 and HYAL3 are clustered on chromosome 3p21.3, while HYAL4, PHYAL1 and SPAMI are clustered on 7q31.3 (31,32). Notably, HYAL1 and HYAL2 are the major HYALs expressed in human somatic tissues (33). HYAL2 is linked to the plasma membrane by a glycosylphosphatidylinositol anchor (34). HYAL2 initially cleaves HMW-HA to $\sim 20 \mathrm{kDa}$ fragments in cooperation with CD44 (35-37). The levels of HYAL2 expression are correlated with the invasive potential of human breast cancer cells $(38,39)$. Furthermore, Udabage et al (39) recently revealed that highly invasive breast cancer cell lines preferentially express HAS2, HYAL2 and CD44, while less invasive cells express HAS3 and HYAL3. Nevertheless, it has remained controversial whether HYAL2 functions as an oncogene or a tumor suppressor gene (33).

Metastasis, referring to the spread of malignant cells from a primary tumor to distant sites, poses the biggest problem in cancer treatment and is the main cause of death of cancer patients. Cell motility is a principal requirement for tumor cells to undergo invasion during metastasis. There are three types of cell motility, namely random cell motility (chemokinesis), directional cell motility (chemotaxis) and haptotaxis. Chemokinesis is important in the initiation and maintenance of cell migration (40). Furthermore, chemokinesis may play a role during the EMT process, thereby facilitating the separation of tumor cells from their primary site (41). Although HA has previously been reported to induce tumor cell motility, the molecular mechanism underlying the role of HA in chemokinesis during tumor progression remains unclear. The objective of this study was to determine how alterations in HA turnover can affect the induction of chemokinesis in tumor cells. In this study, we examined the potential role of the HA synthesis/ degradation system in tumor cell motility and found that the HAS2-HYAL2/CD44 system supports chemokinesis stimulated by fragmented HA on the plasma membrane in an autocrine manner.

\section{Materials and methods}

Materials. A human HYAL2 cDNA clone (GenBank accession no.: NM_033158.2) in pCMV6-XL4 was purchased from OriGene (Rockville, MD). The empty pCMV6-XL4 vector was used as a control. An anti-HYAL2 polyclonal antibody was raised in a rabbit against the amino acid sequence CFYRDRLGLYPRFDSAGRSV. The obtained antibody was affinity-purified using the immunizing peptide. An anti-CD44 monoclonal antibody, Hermes 3 , was purified from conditioned medium of hybridoma HB-9480 cells purchased from the American Type Culture Collection (ATCC, Rockville, MD) (10). An anti-HAS2 antibody (Y-14; sc-34068) was obtained from Santa Cruz Biotechnology (Santa Cruz,CA). An anti-actin antibody (A 2066) was purchased from Sigma (St. Louis, MO). One type of purified LMW-HA fragments and two types of HMW-HA from chicken comb were obtained from Seikagaku Co. (Tokyo, Japan). These HAs were free of protein and other glycosaminoglycans and had peak molecular weights of 23 , 230 and $920 \mathrm{kDa}$, respectively. The HA fragments had an endotoxin content of $<0.002 \mathrm{ng} / \mathrm{mg}$ as determined by Limulus amebocyte lysate assays. 3-(4,5-Dimethyl-2-thiazolyl)-2,5-
diphenyl-2H-tetrazolium bromide for the MTT assay obtained from Sigma.

Cell culture. A human cervical cancer cell line, HeLa-S3, and a human breast cancer cell line, MDA-MB-231, were obtained from the ATCC. HeLa-S3 cells were cultured in DMEM (Nissui, Tokyo, Japan) supplemented with $10 \%$ heat-inactivated FCS and $4 \mathrm{mM}$ L-glutamine. MDA-MB-231 cells were cultured in DMEM supplemented with $10 \%$ heat-inactivated FBS and $4 \mathrm{mM} \mathrm{L}$-glutamine. Both cell lines were maintained at $37^{\circ} \mathrm{C}$ in a $5 \% \mathrm{CO}_{2}$ incubator.

Western blotting. Whole cell lysates were prepared with icecold RIPA buffer (50 mM Tris-HCl pH 7.5, $1 \%$ nonidet P-40, $150 \mathrm{mM} \mathrm{NaCl}, 0.1 \%$ SDS, $0.5 \%$ deoxycholic acid) containing $1 \mu \mathrm{g} / \mathrm{ml}$ leupeptin, $1 \mu \mathrm{g} / \mathrm{ml}$ pepstatin, $1 \mu \mathrm{g} / \mathrm{ml}$ aprotinin, $1 \mathrm{mM}$ DTT, $1 \mathrm{mM} \mathrm{NaVO}_{4}$ and $0.5 \mathrm{mM} \mathrm{PMSF}$. The supernatants were saved as total cell lysates following centrifugation. Aliquots of the cell lysates $(50 \mu \mathrm{g}$ of protein) were separated by $10 \%$ SDS-PAGE and transferred to PVDF membranes (Bio-Rad Laboratories, Hercules, CA). Primary antibodies that bound to their antigens on the membranes were detected using appropriate HRP-conjugated secondary antibodies (Amersham Bioscience, Piscataway, NJ) and a Super Signal chemiluminescence detection system (Pierce, Rockford, IL) or the Lumi-Light ${ }^{\mathrm{PLUS}}$ Western blotting substrate (Roche Diagnostics, Basel, Switzerland) according to the manufacturer's instructions.

Transfection with expression vectors and siRNAs. Expression vectors and siRNAs were introduced into cells using Lipofectamine $^{\mathrm{TM}} 2000$ (Invitrogen, Carlsbad, CA) according to the manufacturer's instructions. Stealth ${ }^{\mathrm{TM}}$ siRNAs specific for HYAL2 (sense, 5'-AAUAUUGGGUGGCCCAGGACAC AUU-3'; antisense, 5'- AAUGUGUCCUGGGCCACCCAAU AUU-3'), HAS2 (sense, 5'-UUACCAUCUCCACAGAUGAG GCUGG-3'; antisense, 5'-CCAGCCUCAUCUGUGGAGAU GGUAA-3') and CD44 (sense, 5'-UAUAUUCAAAUCGAUC UGCGCCAGG-3'; antisense, 5'-CCUGGCGCAGAUCGA UUUGAAUAUA-3') were purchased from Invitrogen. A scramble stealth siRNA (sense, 5'-UAUCCAAC UUCUAACU AGGCGUAGG-3'; antisense 5'-CCUACGCCUAGUUAGA AGUUGGAUA- $3^{\prime}$ ) used as a control, and was also purchased from Invitrogen. A siRNA against HAS3 (sc-45295) and the corresponding scramble control siRNA (sc-37007) were obtained from Santa Cruz Biotechnology.

Real-time RT-PCR. Total RNA was extracted from cells using the TRIzol reagent (Invitrogen), and cDNAs were synthesized with a PrimeScript ${ }^{\text {TM }}$ RT reagent kit (Takara Bio, Shiga, Japan) according to the manufacturer's protocols. Real-time PCR was performed using SYBR Premix Ex Taq ${ }^{\mathrm{TM}}$ (Takara Bio) and an ABI Prism 7000 sequence detection system (Applied Biosystems, Foster City, CA) under the following conditions: $30 \mathrm{sec}$ at $95^{\circ} \mathrm{C} ; 40$ cycles of $5 \mathrm{sec}$ at $95^{\circ} \mathrm{C}$ and $31 \mathrm{sec}$ at $60^{\circ} \mathrm{C}$. Specific primer sets for HYAL2 (forward, 5'-ACCATGCAC TCCCAGTCTACGTC-3'; reverse, 5'-TCGCCAATGGTAGA GATGAGGTC-3'), HAS1 (forward, 5'-AGCAGCCTGCGATA CTGGGTAG-3'; reverse, 5'-CTGGTTGTACCAGGCCTCAA GAA-3'), HAS2 (forward, 5'-TATCACTGCTGCTGGGACTT GAAC-3'; reverse, 5'-A ACACTTTCAGGCGGATGCAC-3'), 
HAS3 (forward, 5'-TCGGCGATTCGGTGGACTA-3'; reverse, 5'-CCTCCAGGACTCGAAGCATCTC-3') and GAPDH (forward, 5'-GCACCGTCAAGGCTGAGAAC-3'; reverse, 5'-TGGTGAAGACGCCAGTGGA-3') were purchased from Takara Bio. The specificity of each amplification was confirmed by a dissociation curve consisting of a single peak. All samples were amplified in triplicate in each experiment. The values were normalized by the expression of GAPDH.

Boyden chamber assay. To investigate the roles of HYAL2 and HAS2 in spontaneous chemokinesis, we employed the Boyden chamber migration assay (42). Expression vectors and siRNAs were transfected into HeLa-S3 or MDA-MB-231 cells using Lipofectamine $^{\text {TM }} 2000$ (Invitrogen). After incubation for $30 \mathrm{~h}$, the cells were starved overnight in DMEM supplemented with $2 \%$ CS or FBS. The cells were then harvested and subjected to Boyden chamber assays. HeLa-S3 cells $\left(5 \times 10^{4}\right.$ cells/well $)$ or MDA-MB-231 cells $\left(2.5 \times 10^{4}\right.$ cells/well) were loaded in the upper chambers of polycarbonate membrane transwell inserts (Corning Inc., Corning, NY). Each set of lower and upper chambers was separated by an $8-\mu \mathrm{m}$ pore size polycarbonate membrane. To evaluate the chemokinesis, each upper and lower chamber contained the same concentration of serum ( $2 \%$ CS for HeLa-S3 cells and 2\% FBS for MDA-MB-231 cells) or $50 \mu \mathrm{g} / \mathrm{ml}$ of HA $(23,230$ and $920 \mathrm{kDa})$. The cells were allowed to migrate for 4 or $6 \mathrm{~h}$. The membranes were then fixed with formalin and stained with Meyer's hematoxylin. The cells on the upper side of the membranes were removed with cotton swabs. The cells on the lower side of the membranes were counted under a light microscope at x 200 magnification. Four random microscopic fields were counted.

Gel permeation chromatography assay. To determine the molecular weights of HA in the conditioned medium, gel permeation chromatography was performed as previously described (43). Briefly, a $100-\mu 1$ aliquot of concentrated and desalted conditioned medium was loaded onto a column system consisting of serially connected TSK-G4000PW $\mathrm{XL}_{\mathrm{XL}}$ and $\mathrm{G} 3000 \mathrm{PW}_{\mathrm{XL}}$ columns (Tosoh Corporation, Tokyo, Japan). The columns were eluted with $0.2 \mathrm{M} \mathrm{NaCl}$ at a flow rate of $0.5 \mathrm{ml} / \mathrm{min}$, and the eluted HA was monitored by measuring the UV absorbance at $215 \mathrm{~nm}$. A calibration curve was created using molecular weight standards for HA (2310, 1410, 993, 847, 637, 460, 104 and $18 \mathrm{kDa}$ ).

MTT assay. The MTT assay, which is widely used to measure cell proliferation and screen for anticancer drugs, is based on the reduction of a tetrazolium salt (44-46). We used the MTT assay to evaluate the effects of the level of HYAL2 expression on cell proliferation. HeLa-S3 cells transfected with HYAL2 expression vector/empty vector or siRNAs for HYAL2/ scramble control were plated in 96-well plates at $1.5 \times 10^{4}$ and $2.5 \times 10^{4}$ cells/well, respectively. At each time point (days $0-4$ ), $20 \mu 1$ of MTT reagent was added and the plates were incubated for $4 \mathrm{~h}$. Subsequently, $100 \mu 1$ of $10 \%$ SDS and $0.01 \mathrm{~N} \mathrm{HCl}$ were added to each well. After overnight incubation, the absorbances were measured at $570 \mathrm{~nm}$ using a Vmax microplate reader (Molecular Devices, Tokyo, Japan). In these experiments, five replicate wells were used for each time point, and the results were calculated as the mean $\pm \mathrm{SD}$.

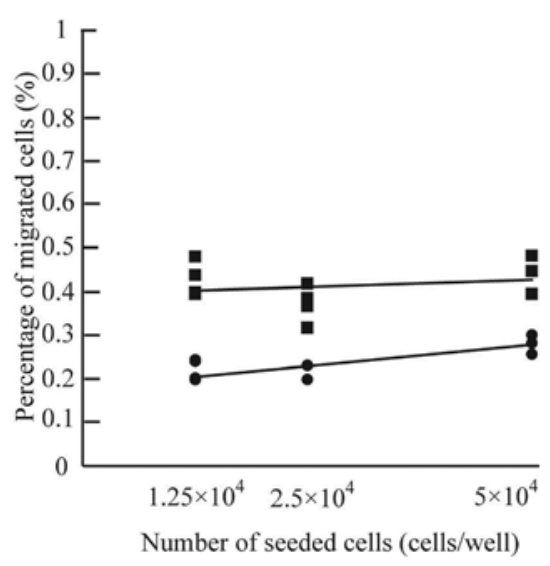

Figure 1. HeLa-S3 cells regulate spontaneous chemokinesis in a cell-autonomous manner. After 4 or $6 \mathrm{~h}$ of incubation, the differences in the spontaneous chemokinesis of HeLa-S3 cells seeded onto the upper chambers at $1.25 \times 10^{4}$, $2.5 \times 10^{4}$ or $5 \times 10^{4}$ cells/well were analyzed by Boyden chamber assays. Closed circles, incubation time of $4 \mathrm{~h}$; closed squares, incubation time of $6 \mathrm{~h}$. The correlation coefficients were obtained by linear regression analyses: $4 \mathrm{~h}, \mathrm{r}=0.986$, $\mathrm{p}<0.001 ; 6 \mathrm{~h}, \mathrm{r}=0.99$, $\mathrm{p}<0.001(\mathrm{n}=4)$.

Statistical analysis. The relationship between the density of plated cells and spontaneous chemokinesis was determined by a linear regression analysis. The statistical significance of differences in spontaneous chemokinesis was determined by Student's t-test. Values of $p<0.05$ were considered to indicate statistical significance.

\section{Results}

Chemokinesis of HeLa-S3 cells is driven by cell-intrinsic forces. In the course of our studies on the effects of HA on epithelial cell motility, we observed spontaneous random cell movement of HeLa-S3 cells in medium with a relatively low serum concentration (2\%), and termed this random cell motility spontaneous chemokinesis. We investigated the effects of spontaneous chemokinesis by seeding different numbers of cells in the upper wells of a Boyden chamber system. We found that the percentages of cells undergoing chemokinesis were not affected by the number of cells at either 4 or $6 \mathrm{~h}$ (Fig. 1). These findings indicate that the spontaneous chemokinesis is mediated by mechanisms intrinsic to individual cells and suggest an autocrine pathway.

HAS enzymes regulate the spontaneous chemokinesis of $\mathrm{HeLa}-\mathrm{S} 3$ cells. In somatic tissues, HA is synthesized by three distinct HAS enzymes, HAS1, HAS2 and HAS3 $(14,15)$. To assess the role of HA production in the spontaneous chemokinesis, we examined the mRNA expression levels of the three HAS enzymes in HeLa-S3 cells by real-time RT-PCR. As shown in Fig. 2A, HAS1 mRNA expression was undetectable in HeLa-S3 cells, although it was detected in HT-1376 human bladder cancer cells as a positive control (19). In contrast, HeLa-S3 cells mainly expressed HAS2 and HAS3 mRNAs (see below). Therefore, we tried to knockdown the expression of HAS2 or HAS3 in HeLa-S3 cells by RNAi. As shown in Fig. 2B, transient transfection of a siRNA directed against the HAS2 mRNA decreased the HAS2 protein expression compared with 

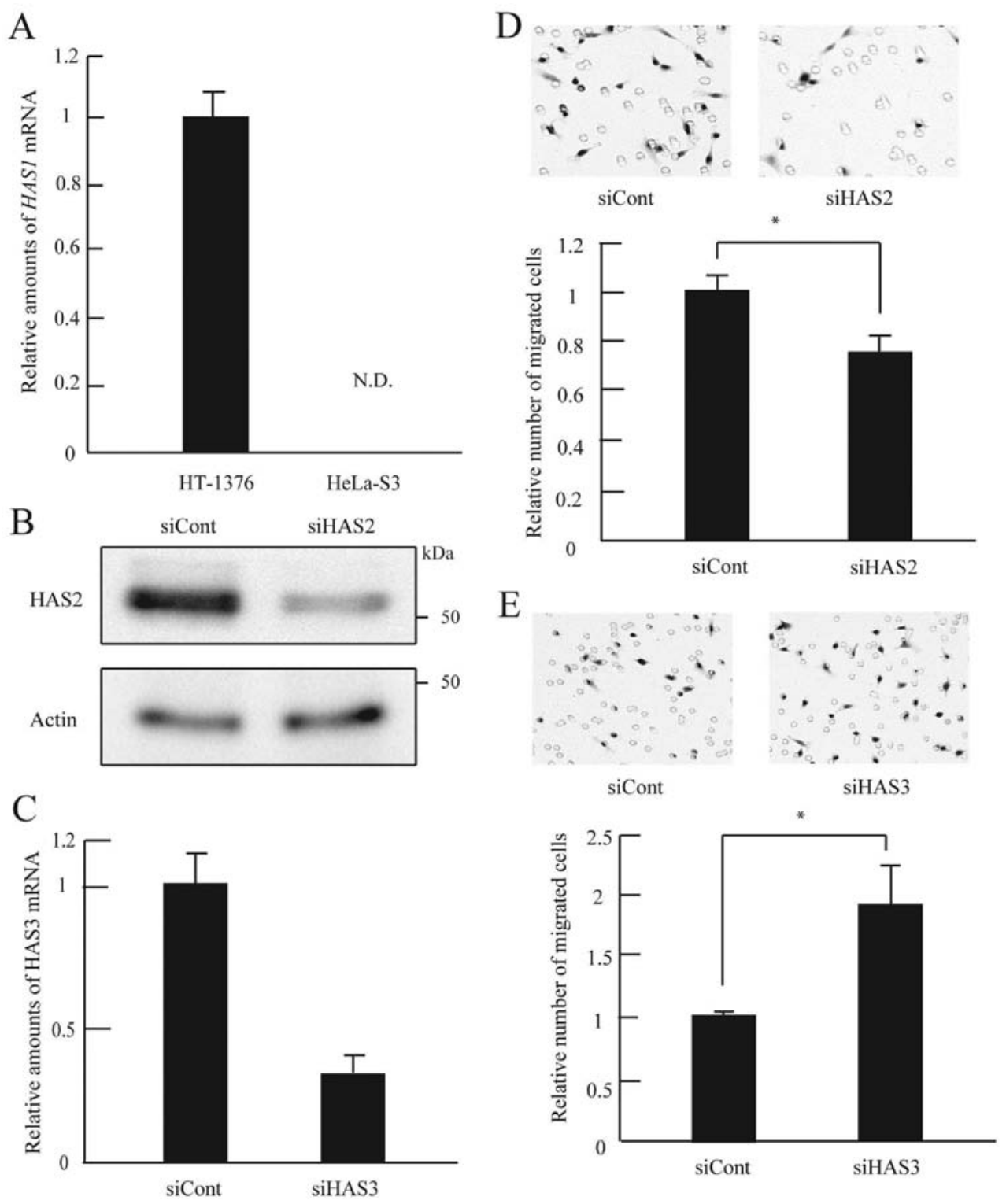

Figure 2. Endogenous HA produced by HAS2 regulates the spontaneous chemokinesis of HeLa-S3 cells. (A) The mRNA expression of HAS1 was measured in HeLa-S3 and HT-1376 cells by real-time RT-PCR. HT-1376 cells were evaluated as a positive control to verify the primer specificity. The data shown are means \pm SD $(n=3)$. (B) Western blotting of HAS2 protein in HeLa-S3 cells transfected with the control (siCont) or HAS2 (siHAS2) siRNAs. (C) Real-time RT-PCR analysis of HAS3 mRNA expression in HeLa-S3 cells transfected with the control (siCont) or HAS3 (siHAS3) siRNAs. The data shown are means \pm $\mathrm{SD}(\mathrm{n}=3)$. (D) The effect of the siHAS2 siRNA on spontaneous chemokinesis of HeLa-S3 cells was assessed by Boyden chamber assays. Representative images of a migration assay for migrated HeLa-S3 cells transfected with the siCont or siHAS2 siRNAs are shown. Magnification, x200. The data shown are means $\pm \mathrm{SD}(\mathrm{n}=4) .{ }^{*} \mathrm{p}<0.01$, vs. the control by Student's t-test. (E) The effect of the siHAS3 siRNA on spontaneous chemokinesis of HeLa-S3 cells was assessed by Boyden chamber assays. Representative images of a migration assay for migrated HeLa-S3 cells transfected with the siCont or siHAS3 siRNAs are shown. Magnification, $x 200$. The data shown are means \pm SD $(n=4) .{ }^{*} p<0.01$, vs. the control by Student's t-test.

cells transfected with a scramble control siRNA. Similarly, we were able to reduce the HAS3 mRNA expression by siRNA-mediated knockdown (Fig. 2C). When we assessed spontaneous chemokinesis of the cells transfected with the HAS2 siRNA, we found that the cell motility was significantly reduced compared with the control siRNA-transfected cells (Fig. 2D). Both knockdown of HAS2 expression and reduction of spontaneous chemokinesis were induced by siRNAs with independent sequences derived from the HAS2 mRNA (data not shown). In contrast, knockdown of HAS3 mRNA expression increased spontaneous chemokinesis of HeLa-S3 cells (Fig. 2E). In agreement with a previous study performed with a different cell line (17), knockdown of HAS2 mRNA expression in HeLa-S3 cells induced a remarkable increase in HAS3 mRNA expression (Fig. 3A), while knockdown of HAS3 mRNA expression caused a significant elevation of HAS2 mRNA expression (Fig. 3B). These results indicate that the expression level of HAS2 is involved in the spontaneous chemokinesis of HeLa-S3 cells.

Cell-associated HA plays a critical role in the regulation of spontaneous chemokinesis. To further investigate the roles of the HAS enzymes in the spontaneous chemokinesis, we determined the molecular weights of HA produced by HeLa-S3 
A
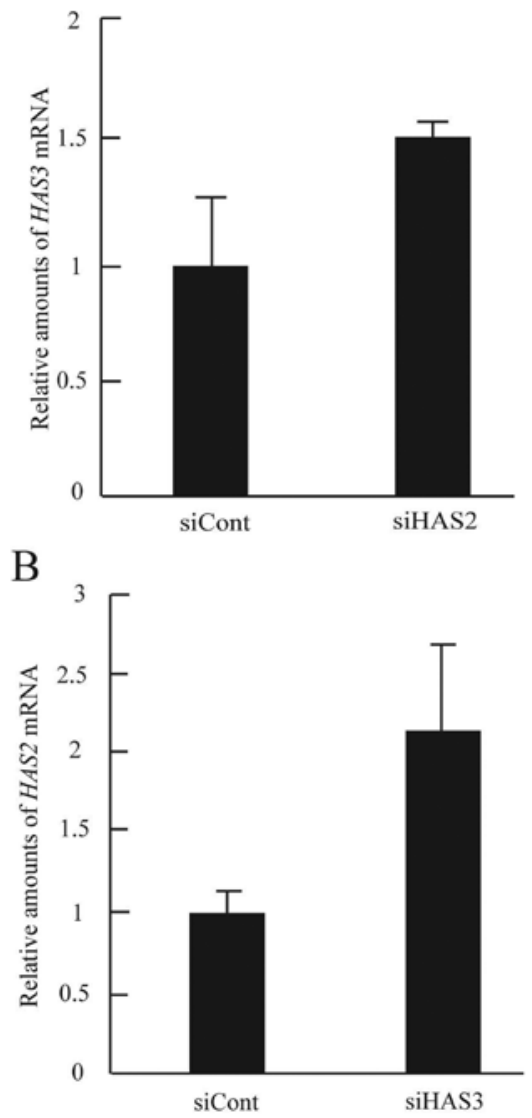

Figure 3. Knockdown of HAS2 mRNA expression in HeLa-S3 cells induces a remarkable increase in $H A S 3$ mRNA expression, while knockdown of $H A S 3$ mRNA expression causes a significant elevation of HAS 2 mRNA expression. (A) Real-time RT-PCR analysis of HAS3 mRNA expression in HeLa-S3 cells treated with the control (siCont) or HAS2 (siHAS2) siRNAs. The data shown are means $\pm \mathrm{SD}(\mathrm{n}=3)$. (B) Real-time RT-PCR analysis of HAS2 mRNA expression in HeLa-S3 cells transfected with the control (siCont) or HAS3 (siHAS3) siRNAs. The data shown are means $\pm \mathrm{SD}(\mathrm{n}=3)$.

cells by gel permeation chromatography assays. HeLa-S3 cells cultured in serum-free medium for $6 \mathrm{~h}$ produced $\sim 50 \mathrm{ng} /$ $\mathrm{ml}$ of HA with a peak molecular mass of $990 \mathrm{kDa}$ (Fig. 4A). These results are consistent with the notion that the HA is generated by HAS2, which produces HMW-HA in HeLa-S3 cells. Since the expression of HAS2 appeared to be correlated with the cell motility of HeLa-S3 cells, we assessed the effects of an excessive amount of the $920 \mathrm{kDa} H A$ on the spontaneous chemokinesis of the cells. However, exogenously added $920 \mathrm{kDa} H A$ did not enhance the spontaneous chemokinesis of the cells within $6 \mathrm{~h}$ (Fig. 4B). These findings suggest that the HMW-HA, which is presumably generated by HAS2, does not directly induce the spontaneous chemokinesis of HeLa-S3 cells. The findings further indicate that HeLa-S3 cells may have a self-degrading system for HA to regulate their spontaneous chemokinesis.

Knockdown of HYAL2 or CD44 reduces the spontaneous chemokinesis of HeLa-S3 cells. It was previously shown that HMW-HA bound to the cell surface with the aid of CD44 is preferentially degraded during a culture period of 2-6 $\mathrm{h}$ in HEK293 cells overexpressing both HYAL2 and CD44 (36).
A
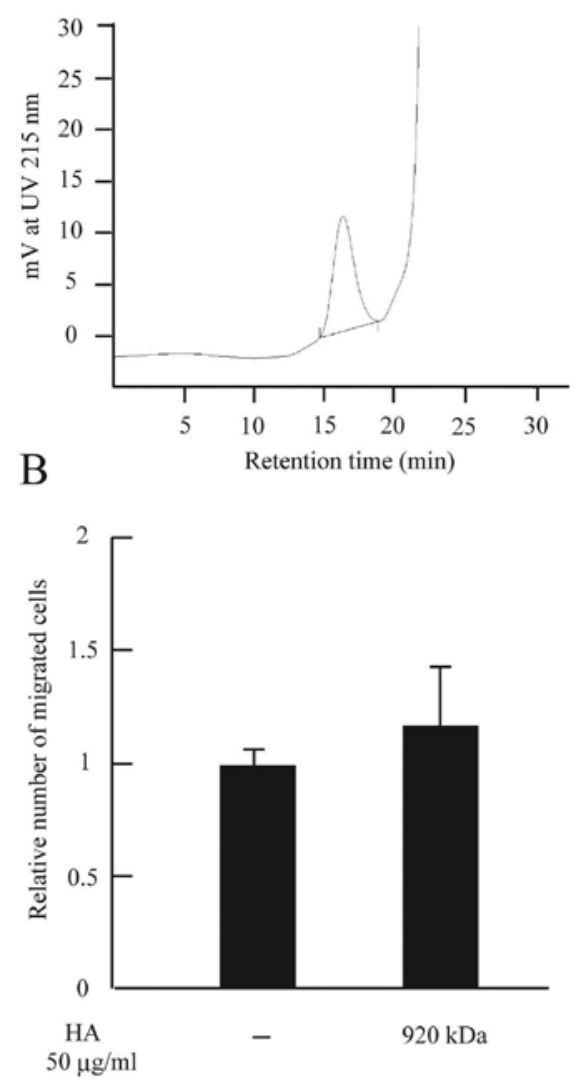

Figure 4. Cell-associated HMW-HA plays a critical role in the regulation of spontaneous chemokinesis. (A) Gel permeation chromatography profile of HA produced by HeLa-S3 cells. (B) The effect of the $920 \mathrm{kDa}$ HA on spontaneous chemokinesis of HeLa-S3 cells was assessed by Boyden chamber assays. The data shown are means $\pm \operatorname{SD}(n=4)$.

Accordingly, our results indicated that the HA generated by HAS2 in HeLa-S3 cells may need to be degraded by HYAL2 at the cell surface to regulate spontaneous chemokinesis. To evaluate the effects of HYAL2 on spontaneous chemokinesis, we performed siRNA-mediated knockdown of HYAL2 in HeLa-S3 cells. In Western blot analyses, an anti-HYAL2 antibody recognized two bands with apparent molecular masses of $\sim 54 \mathrm{kDa}$ (Fig. 5A), as expected from the structure of the HYAL2 cDNA. Introduction of the HYAL2 siRNA, but not the control siRNA or an unrelated siRNA, resulted in almost complete reduction of the slower migrating band but not the faster migrating band (Fig. 5A). Unfortunately, we had a nonspecific band near HYAL2 band by Western blot analysis. It did not change by the treatment of HYAL2 siRNA. As shown in Fig. 5B and C, this siRNA treatment did not change the mRNA expression levels of either HAS2 or HAS3 when measured by real-time RT-PCR. When we assessed spontaneous chemokinesis of the HeLa-S3 cells with reduced expression of HYAL2, we found that the motility was markedly decreased compared with the control siRNA-transfected cells (Fig. 5D). These findings are consistent with the idea that HYAL2 is involved in the HA-dependent spontaneous chemokinesis. Next, to assess the effects of CD44 on spontaneous chemokinesis, we examined siRNA-mediated knockdown of CD44 expression in HeLa-S3 cells. Introduction of a siRNA against CD44 resulted in a 
A

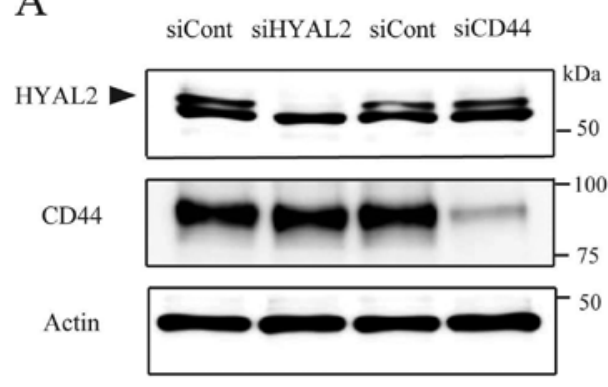

B

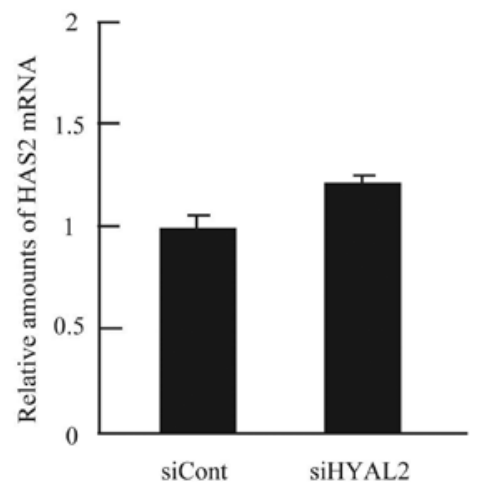

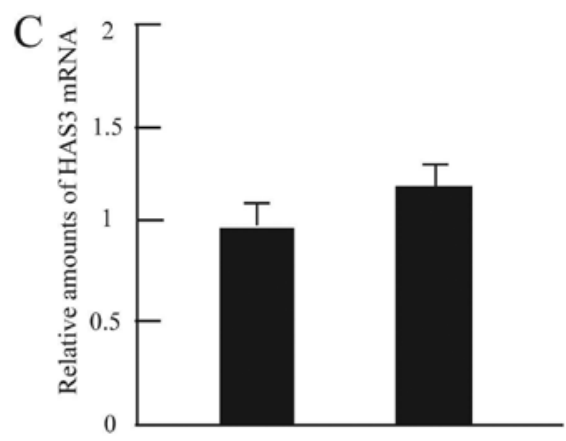

$\mathrm{D}$

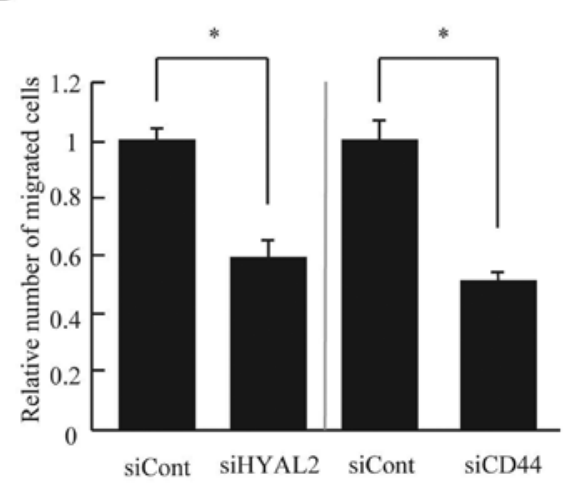

Figure 5. Knockdown of either HYAL2 or CD44 expression reduces spontaneous chemokinesis of HeLa-S3 cells. (A) Western blot analysis of HYAL2 and CD44 in HeLa-S3 cells transfected with the control (siCont), HYAL2 (siHYAL2) or CD44 (siCD44) siRNAs. (B and C) Comparisons of the HAS gene expression levels between HeLa-S3 cells transfected with the siCont and siHYAL2 siRNAs. The mRNA levels of HAS2 (B) and HAS3 (C) were determined by real-time RT-PCR. The data shown are means \pm SD $(n=3)$. (D) Spontaneous chemokinesis was assessed by Boyden chamber assays. Representative images of a migration assay for migrated HeLa-S3 cells transfected with the siCont, siHYAL2 or siCD44 siRNAs are shown. Magnification, x200. The data shown are means $\pm \operatorname{SD}(n=4) .{ }^{*} \mathrm{p}<0.01$, vs. siCont by Student's t-test.
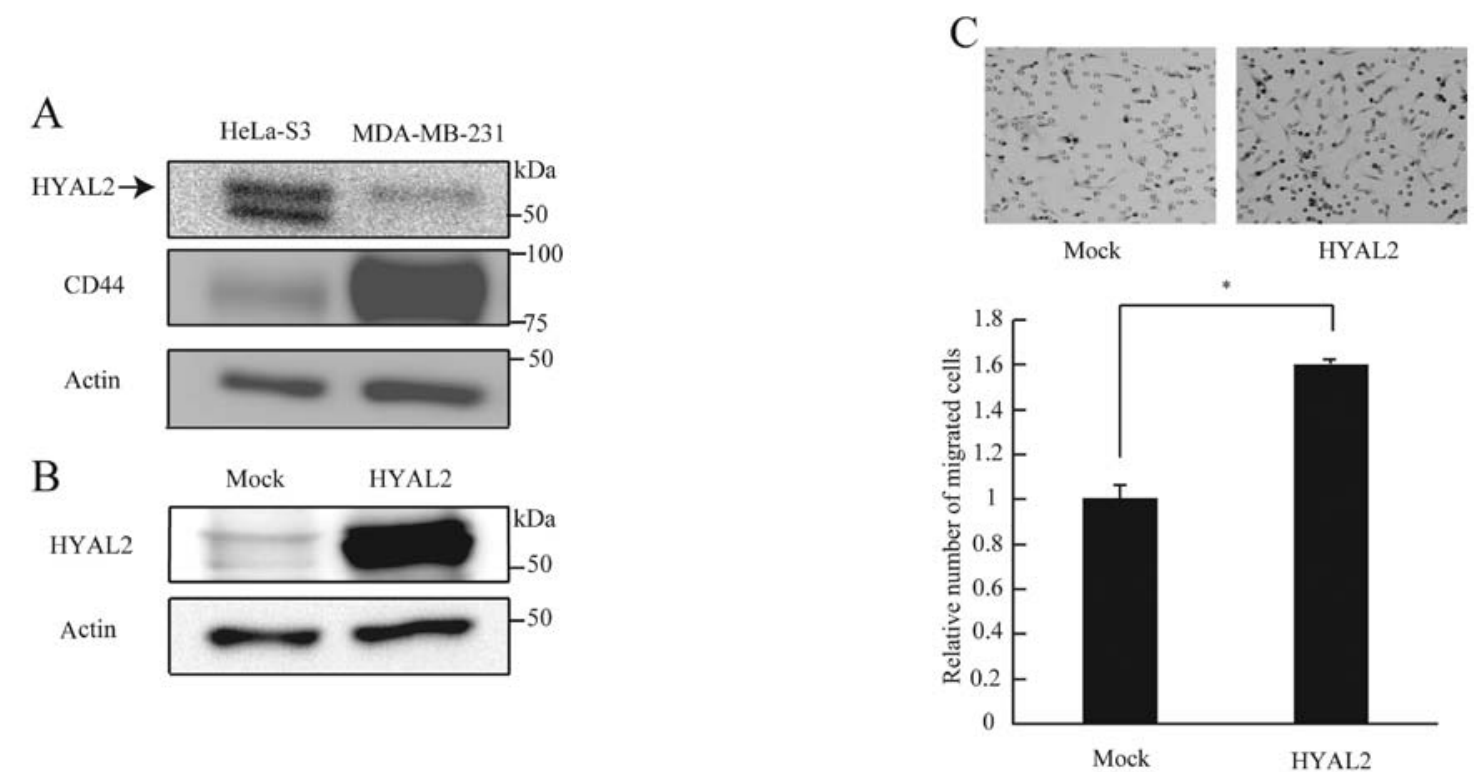

Figure 6. Transient HYAL2 expression enhances spontaneous chemokinesis of MDA-MB-231 cells. (A) Comparison of the HYAL2 protein expression levels in HeLa-S3 and MDA-MB-231 cells by Western blot analysis. (B) Western blot analysis of HYAL2 protein in MDA-MB-231 cells transfected with the empty vector (mock) or HYAL2-expressing vector. (C) The effect of HYAL2 overexpression on spontaneous chemokinesis of MDA-MB-231 cells was analyzed by Boyden chamber assays. Representative images of a migration assay for migrated MDA-MB-231 cells expressing the empty (mock) or HYAL2-expressing vector. Magnification, $x 200$. The data shown are means $\pm \operatorname{SD}(n=4)$. ${ }^{*} p<0.01$, vs. the control by Student's t-test.

remarkable decrease in the protein expression as detected by Western blot analysis (Fig. 5A). This treatment also decreased spontaneous chemokinesis compared with the control siRNAtransfected cells (Fig. 5D). These findings indicate that CD44 
A

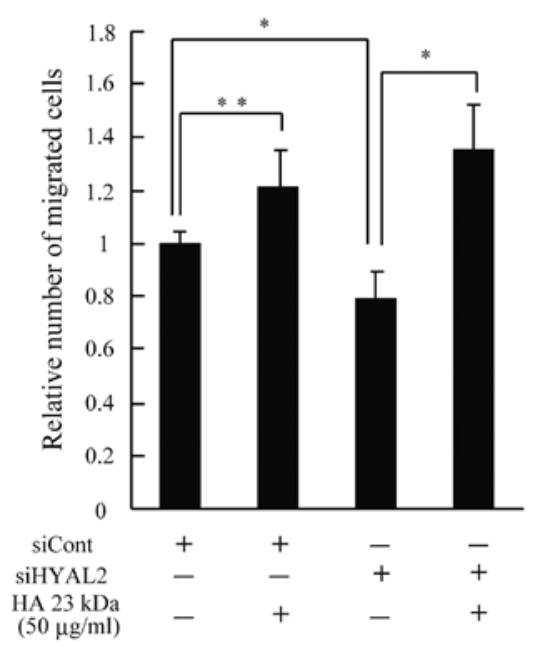

$\mathrm{C}$

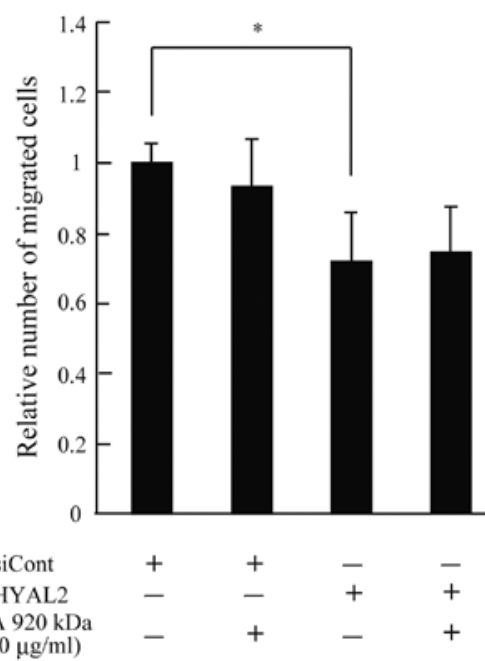

B

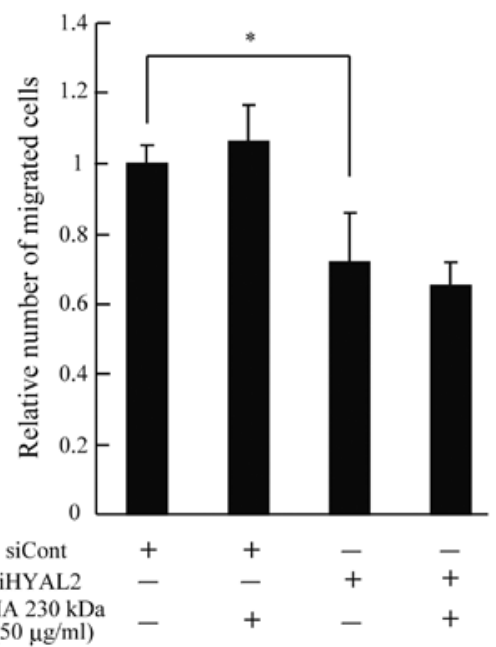

Figure 7. HA ( $23 \mathrm{kDa})$ reverses the siHYAL2-mediated reduction of spontaneous chemokinesis to the control level. The effects of different molecular weight HAs (23, 230 and $920 \mathrm{kDa}$ ) on the HYAL2 siRNA (siHYAL2)-mediated reduction of spontaneous chemokinesis of HeLa-S3 cells were assessed by Boyden chamber assays. (A-C) The siHYAL2-transfected HeLa-S3 cells were incubated in the absence or presence of $50 \mu \mathrm{g} / \mathrm{ml}$ of the $23 \mathrm{kDa} \mathrm{HA}$ (A), $230 \mathrm{kDa} \mathrm{HA}$ (B) or $920 \mathrm{kDa}$ HA (C). The data shown are means $\pm \mathrm{SD}(\mathrm{n}=5)$. ${ }^{*} \mathrm{p}<0.01,{ }^{* *} \mathrm{p}<0.05$, vs. the control by Student's t-test.

is also involved in HA-dependent spontaneous chemokinesis and that catabolism of HA by HYAL2 and CD44 at the cell surface is a key event in the regulation of the spontaneous chemokinesis. To further validate the involvement of HYAL2 in the motility, we examined the MDA-MB-231 cell line, as a CD44-positive breast cancer cell line $(39,47)$, which expressed lower levels of HYAL2 protein than HeLa-S3 cells (Fig. 6A). MDA-MB-231 cells transiently transfected with the HYAL2 cDNA robustly expressed HYAL2 protein (Fig. 6B). The HYAL2-overexpressing MDA-MB-231 cells showed a remarkable increase in spontaneous chemokinesis compared with control mock-transfected cells (Fig. 6C). These findings suggest that HYAL2/CD44 degrades HA and that the resulting fragmented HA stimulates cells to undergo spontaneous chemokinesis.

LMW-HA, but not HMW-HA reverses the HYAL2 siRNAmediated reduction of spontaneous chemokinesis to the level in cells stimulated with the corresponding HA. In this study, we found that the knockdown of HYAL2, HAS2 or CD44 resulted in a decrease in spontaneous chemokinesis of HeLa-S3 cells. To determine which sizes of HA are involved in spontaneous chemokinesis of these cells, we obtained HAs with different molecular weights $(23,230$ and $920 \mathrm{kDa})$ and evaluated how they affected the decrease in spontaneous chemokinesis of HeLa-S3 cells after the siRNA-mediated knockdown of HYAL2. Addition of the $23 \mathrm{kDa}$ HA reversed the HYAL2 siRNA-dependent reduction in spontaneous chemokinesis to the level of control cells stimulated with the same HA (Fig. 7A). However, the two HMW-HAs (230 and $920 \mathrm{kDa})$ had no effect on the reduction in the spontaneous chemokinesis of HeLa-S3 cells after siRNA-mediated knockdown of HYAL2 (Fig. 7B and C). These findings indicate that HeLa-S3 cells autonomously generate HYAL2/CD44-mediated HA fragments, which enhance their spontaneous chemokinesis. Therefore, our findings suggest that the HAS2-HYAL2/CD44 system supports HA fragment-dependent cell motility by an autocrine mechanism. 


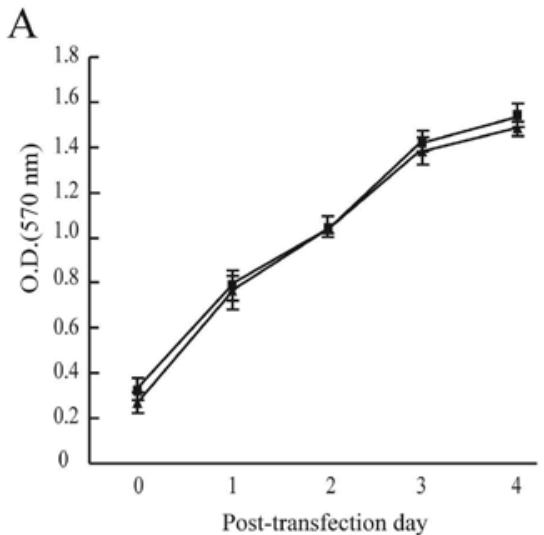

B

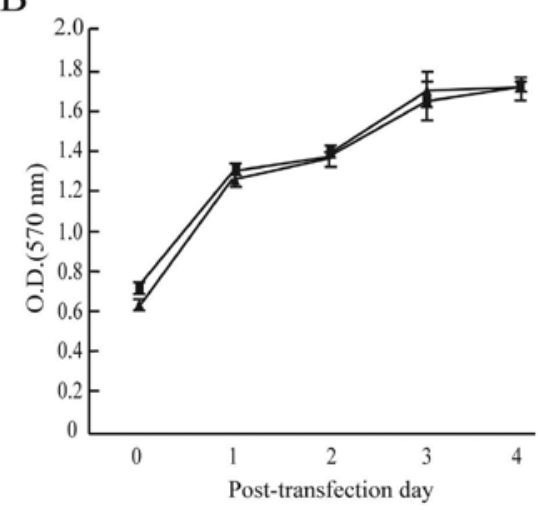

Figure 8. HYAL2 does not affect the proliferation of HeLa-S3 cells. The effects of the HYAL2 expression levels on cell proliferation were analyzed by MTT assays from days 0 to 4 of culture. The data shown are means \pm SD $(\mathrm{n}=5)$. (A) Closed triangles represent cells transfected with the control siRNA and closed squares indicate cells transfected with the HYAL2 siRNA. (B) Closed triangles represent cells transfected with the empty vector and closed squares indicate cells transfected with the HYAL2 vector.

HYAL2 does not affect the proliferation of HeLa-S3 cells. To eliminate the possibility that HYAL2 affected the cell proliferation during the motility assays, we assessed the effects of the alterations in HYAL2 expression on the proliferation of HeLa-S3 cells by MTT assays. As shown in Fig. 8A and B, neither upregulation nor downregulation of HYAL2 expression affected the cell proliferation during a culture period of 4 days. Therefore, the HYAL2-mediated changes in the spontaneous chemokinesis seem to be independent of cell proliferation. These results are consistent with a previous study using different cell lines (48).

\section{Discussion}

$\mathrm{HA}$ is a ubiquitous extracellular matrix component of the tumor environment, especially in the stroma where its accumulation is observed in various carcinomas $(6,49-53)$. In particular, cell-associated HA is found in the plasma membrane of poorly differentiated invasive cells of advanced carcinomas (51). The increased levels of HA indicate that its metabolism is altered in carcinomas, and that this perturbation of HA synthesis and/ or degradation may play important roles in tumor progression. Recently, it has become increasingly clear that LMW-HA can induce a variety of biological events, such as matrix metalloproteinase expression $(54,55)$, angiogenesis (56) and tumor cell migration (57). Our findings suggest that HAS2 synthe- sizes HMW-HA, which is then degraded by the HYAL2/ CD44 complex to LMW-HA on the plasma membrane in an autocrine manner to regulate cancer cell motility.

Previous studies have shown that HAS2 contributes to the initiation and progression of cancer $(17,23-25,29,39)$. Our findings further showed that siRNA-mediated silencing of HAS2 expression decreased the spontaneous chemokinesis of HeLa-S3 cells. This observation supports the notion that HAS2 expression plays important roles in cancer progression and metastasis. We also found that HAS3 appeared to play a minor role in the regulation of the spontaneous chemokinesis of HeLa-S3 cells. Furthermore, the downregulation of HAS3 mRNA expression resulted in increased HAS 2 mRNA expression, and vice versa. These findings suggest the implementation of a back-up cellular mechanism, and these events are likely to occur often in HA-related genes $(17,23)$. HA synthesized by HAS2 may preferentially regulate the cell motility compared with HAS3-synthesized HA in HeLa-S3 cells, since the levels of HAS2 expression but not HAS3 expression were correlated with the rate of enhancement of chemokinesis.

It has remained controversial whether HYAL2 is a tumor promoter or a tumor suppressor. Duterme et al (58) reported that stable overexpression of HYAL2 with no detectable enzymatic activity reduced the cell motility of rat fibroblast cell lines in wound healing assays. In contrast, Udabage et al (39) reported that highly invasive human breast cancer cells preferentially expressed the mRNAs of HAS2, HYAL2 and CD44. Our results support the latter observations that HYAL2 plays a critical role in the increase in cell motility, and that this effect is dependent on the expression levels of HAS2 and CD44. HYAL enzymes and their attendant control systems appear to be of critical importance in the expression of biological HA functions. It was previously shown that HMW-HAs associated with the cell surface are more preferentially degraded during cultures of HEK293 cells overexpressing both HYAL2 and CD44 compared with soluble HMW-HAs in the medium (36). Our findings indicate that the HYAL2/CD44 complex degrades HAS2-synthesized HMW-HA and that the resultant LMW-HA may act on hyaladherin receptors including CD44.

There have been a number of reports regarding CD44 ligation-induced cellular responses, including enhancement of cell motility $(59,60)$, expression of adhesion molecules $(61,62)$ and a growth factor receptor (62), cytokine and chemokine secretion (63-66), and protection from apoptosis (10,67-69). At the biochemical level, CD44 ligation has been shown to activate Racl (70-72) and nuclear factor- $\kappa B$ (73). An emerging concept in signal transduction is that cell adhesion molecules can function as co-receptor (74). CD44 is known to serve as a co-receptor for growth factors and associate with their receptors, such as tyrosine kinase receptor ErbB or c-Met $(75,76)$. Some of these CD44 ligation-induced cellular responses would be related to LMW-HA production by the HAS2-HYAL2/ CD44 system during neoplastic transformation.

The major signaling pathways relevant to cancer are involved in HA metabolism. Essentially, EGF receptor signaling increases HA production via elevation of HAS2 expression (77,78), p53 protein suppresses the expression of hyaladherins, such as CD44 (79), and Wnt signaling regulates the expression of CD44 (80). Accordingly, these lines of evidence, together with the findings of the present study, support the notion that altera- 
tions of HA metabolism in cancer cells could open a gateway for invasion and metastasis. The present findings indicate that HMW-HA synthesized by HAS2 is degraded by the HYAL2/ CD44 complex to $20 \mathrm{kDa}$ LMW-HA on the membrane, which then acts on hyaladherin receptors. Subsequently, when a molecular interaction with an appropriate signaling receptor occurs, the resultant signaling activates Rho GTPases, which induce cell motility through dynamic cytoskeletal reorganization. All of these steps are controlled by the cells. Our study suggests that this autocrine LMW-HA-induced stimulation may be a novel activation mechanism that could facilitate cancer cell motility during epithelial-mesenchymal transition at the invasive front of metastatic cancer.

\section{Acknowledgments}

We thank the Seikagaku Corporation for providing the HA, T. Nakamura (Seikagaku Co.) for assistance with the gel permeation chromatography assays, and T. Oyama, M. Higashi and T. Umemiya for critical discussions. This work was supported in part by a grant-in-aid for Scientific Research on Priority Areas from the Ministry of Education, Culture, Sports, Science and Technology of Japan (15024210 to K.H), Grants-in-Aid for Scientific Research from the Japan Society for the Promotion of Science (15390122 and 22390074 to K.H), and funds from the Department of Molecular and Tumor Pathology, Chiba University Graduate School of Medicine.

\section{References}

1. Toole BP: Hyaluronan: from extracellular glue to pericellular cue. Nat Rev Cancer 4: 528-539, 2004.

2. McBride WH and Bard JB: Hyaluronidase-sensitive halos around adherent cells. Their role in blocking lymphocyte-mediated cytolysis. J Exp Med 149: 507-515, 1979.

3. Feinberg RN and Beebe DC: Hyaluronate in vasculogenesis. Science 220: 1177-1179, 1983.

4. Delmage JM, Powars DR, Jaynes PK and Allerton SE: The selective suppression of immunogenicity by hyaluronic acid. Ann Clin Lab Sci 16: 303-310, 1986.

5. Lokeshwar VB, Obek C, Soloway MS and Block NL: Tumorassociated hyaluronic acid: a new sensitive and specific urine marker for bladder cancer. Cancer Res 57: 773-777, 1997.

6. Lokeshwar VB, Rubinowicz D, Schroeder GL, et al: Stromal and epithelial expression of tumor markers hyaluronic acid and HYAL1 hyaluronidase in prostate cancer. J Biol Chem 276: 11922-11932, 2001.

7. Turley EA, Noble PW and Bourguignon LY: Signaling properties of hyaluronan receptors. J Biol Chem 277: 4589-4592, 2002.

8. Bourguignon LY, Zhu H, Chu A, Iida N, Zhang L and Hung MC: Interaction between the adhesion receptor, CD44, and the oncogene product, p185HER2, promotes human ovarian tumor cell activation. J Biol Chem 272: 27913-27918, 1997.

9. Ouhtit A, Abd Elmageed ZY, Abdraboh ME, Lioe TF and Raj MH: In vivo evidence for the role of CD44s in promoting breast cancer metastasis to the liver. Am J Pathol 171: 2033-2039, 2007.

10. Fujita $Y$, Kitagawa M, Nakamura S, et al: CD44 signaling through focal adhesion kinase and its anti-apoptotic effect. FEBS Lett 528: 101-108, 2002.

11. Naor D, Nedvetzki S, Golan I, Melnik L and Faitelson Y: CD44 in cancer. Crit Rev Clin Lab Sci 39: 527-579, 2002.

12. Lokeshwar VB and Selzer MG: Hyalurondiase: both a tumor promoter and suppressor. Semin Cancer Biol 18: 281-287, 2008.

13. Toole BP: Hyaluronan-CD44 interactions in cancer: paradoxes and possibilities. Clin Cancer Res 15: 7462-7468, 2009.

14. Itano N, Sawai T, Yoshida M, et al: Three isoforms of mammalian hyaluronan synthases have distinct enzymatic properties. J Biol Chem 274: 25085-25092, 1999.
15. Weigel PH, Hascall VC and Tammi M: Hyaluronan synthases. J Biol Chem 272: 13997-14000, 1997.

16. Bharadwaj AG, Rector K and Simpson MA: Inducible hyaluronan production reveals differential effects on prostate tumor cell growth and tumor angiogenesis. J Biol Chem 282: 20561-20572, 2007.

17. Li Y, Li L, Brown TJ and Heldin P: Silencing of hyaluronan synthase 2 suppresses the malignant phenotype of invasive breast cancer cells. Int J Cancer 120: 2557-2567, 2007.

18. Itano $\mathrm{N}$ and Kimata $\mathrm{K}$ : Altered hyaluronan biosynthesis in cancer progression. Semin Cancer Biol 18: 268-274, 2008.

19. Golshani R, Lopez L, Estrella V, Kramer M, Iida N and Lokeshwar VB: Hyaluronic acid synthase-1 expression regulates bladder cancer growth, invasion, and angiogenesis through CD44. Cancer Res 68: 483-491, 2008.

20. Itano N, Sawai T, Atsumi F, et al: Selective expression and functional characteristics of three mammalian hyaluronan synthases in oncogenic malignant transformation. J Biol Chem 279: 18679-18687, 2004.

21. Bharadwaj AG, Kovar JL, Loughman E, Elowsky C, Oakley GG and Simpson MA: Spontaneous metastasis of prostate cancer is promoted by excess hyaluronan synthesis and processing. Am J Pathol 174: 1027-1036, 2009.

22. Simpson MA, Wilson CM and McCarthy JB: Inhibition of prostate tumor cell hyaluronan synthesis impairs subcutaneous growth and vascularization in immunocompromised mice. Am J Pathol 161: 849-857, 2002.

23. Udabage L, Brownlee GR, Waltham M, et al: Antisense-mediated suppression of hyaluronan synthase 2 inhibits the tumorigenesis and progression of breast cancer. Cancer Res 65: 6139-6150, 2005.

24. Kosaki R, Watanabe K and Yamaguchi Y: Overproduction of hyaluronan by expression of the hyaluronan synthase Has2 enhances anchorage-independent growth and tumorigenicity. Cancer Res 59: 1141-1145, 1999.

25. Nishida Y, Knudson W, Knudson CB and Ishiguro N: Antisense inhibition of hyaluronan synthase- 2 in human osteosarcoma cells inhibits hyaluronan retention and tumorigenicity. Exp Cell Res 307: 194-203, 2005.

26. Camenisch TD, Schroeder JA, Bradley J, Klewer SE and McDonald JA: Heart-valve mesenchyme formation is dependent on hyaluronan-augmented activation of ErbB2-ErbB3 receptors. Nat Med 8: 850-855, 2002.

27. Camenisch TD, Spicer AP, Brehm-Gibson T, et al: Disruption of hyaluronan synthase-2 abrogates normal cardiac morphogenesis and hyaluronan-mediated transformation of epithelium to mesenchyme. J Clin Invest 106: 349-360, 2000.

28. Rodgers LS, Lalani S, Hardy KM, et al: Depolymerized hyaluronan induces vascular endothelial growth factor, a negative regulator of developmental epithelial-to-mesenchymal transformation. Circ Res 99: 583-589, 2006.

29. Zoltan-Jones A, Huang L, Ghatak S and Toole BP: Elevated hyaluronan production induces mesenchymal and transformed properties in epithelial cells. J Biol Chem 278: 45801-45810, 2003.

30. Jenkins RH, Thomas GJ, Williams JD and Steadman R: Myofibroblastic differentiation leads to hyaluronan accumulation through reduced hyaluronan turnover. J Biol Chem 279: 4145341460, 2004.

31. Csoka AB, Frost GI and Stern R: The six hyaluronidase-like genes in the human and mouse genomes. Matrix Biol 20: 499-508, 2001.

32. Csoka AB, Scherer SW and Stern R: Expression analysis of six paralogous human hyaluronidase genes clustered on chromosomes 3p21 and 7q31. Genomics 60: 356-361, 1999.

33. Stern R: Devising a pathway for hyaluronan catabolism: are we there yet? Glycobiology 13: R105-R115, 2003.

34. Rai SK, Duh FM, Vigdorovich V, Danilkovitch-Miagkova A, Lerman MI and Miller AD: Candidate tumor suppressor HYAL2 is a glycosylphosphatidylinositol (GPI)-anchored cell-surface receptor for jaagsiekte sheep retrovirus, the envelope protein of which mediates oncogenic transformation. Proc Natl Acad Sci USA 98: 4443-4448, 2001.

35. Lepperdinger G, Strobl B and Kreil G: HYAL2, a human gene expressed in many cells, encodes a lysosomal hyaluronidase with a novel type of specificity. J Biol Chem 273: 22466-22470, 1998.

36. Harada $\mathrm{H}$ and Takahashi M: CD44-dependent intracellular and extracellular catabolism of hyaluronic acid by hyaluronidase-1 and -2. J Biol Chem 282: 5597-5607, 2007. 
37. de la Motte C, Nigro J, Vasanji A, et al: Platelet-derived hyaluronidase 2 cleaves hyaluronan into fragments that trigger monocyte-mediated production of proinflammatory cytokines. Am J Pathol 174: 2254-2264, 2009.

38. Bourguignon LY, Singleton PA, Diedrich F, Stern R and Gilad E: CD44 interaction with $\mathrm{Na}^{+}-\mathrm{H}^{+}$exchanger (NHE1) creates acidic microenvironments leading to hyaluronidase-2 and cathepsin B activation and breast tumor cell invasion. J Biol Chem 279: 26991-27007, 2004.

39. Udabage L, Brownlee GR, Nilsson SK and Brown TJ: The over-expression of HAS2, Hyal-2 and CD44 is implicated in the invasiveness of breast cancer. Exp Cell Res 310: 205-217, 2005.

40. Hu M, Pollock RE and Nicolson GL: Purification and characterization of human lung fibroblast motility-stimulating factor for human soft tissue sarcoma cells: identification as an NH2-terminal fragment of human fibronectin. Cancer Res 57 3577-3584, 1997.

41. Liotta LA, Mandler R, Murano G, et al: Tumor cell autocrine motility factor. Proc Natl Acad Sci USA 83: 3302-3306, 1986.

42. Toyoda A, Kawana H, Azuhata K, et al: Aberrant expression of human ortholog of mammalian enabled (hMena) in human colorectal carcinomas: implications for its role in tumor progression. Int J Oncol 34: 53-60, 2009.

43. Shimabukuro Y, Ichikawa T, Takayama S, et al: Fibroblast growth factor- 2 regulates the synthesis of hyaluronan by human periodontal ligament cells. J Cell Physiol 203: 557-563, 2005.

44. Hansen MB, Nielsen SE and Berg K: Re-examination and further development of a precise and rapid dye method for measuring cell growth/cell kill. J Immunol Methods 119: 203-210, 1989.

45. Qiang YW, Kitagawa M, Higashi M, Ishii G, Morimoto C and Harigaya K: Activation of mitogen-activated protein kinase through alpha5/beta1 integrin is required for cell cycle progression of B progenitor cell line, Reh, on human marrow stromal cells. Exp Hematol 28: 1147-1157, 2000.

46. Twentyman PR and Luscombe M: A study of some variables in a tetrazolium dye (MTT) based assay for cell growth and chemosensitivity. Br J Cancer 56: 279-285, 1987.

47. Draffin JE, McFarlane S, Hill A, Johnston PG and Waugh DJ: CD44 potentiates the adherence of metastatic prostate and breast cancer cells to bone marrow endothelial cells. Cancer Res 64: 5702-5711, 2004.

48. Wang F, Grigorieva EV, Li J, et al: HYAL1 and HYAL2 inhibit tumour growth in vivo but not in vitro. PLoS One 3: e3031, 2008

49. Lipponen P, Aaltomaa S, Tammi R, Tammi M, Agren U and Kosma VM: High stromal hyaluronan level is associated with poor differentiation and metastasis in prostate cancer. Eur J Cancer 37: 849-856, 2001.

50. Anttila MA, Tammi RH, Tammi MI, Syrjanen KJ, Saarikoski SV and Kosma VM: High levels of stromal hyaluronan predict poor disease outcome in epithelial ovarian cancer. Cancer Res 60: 150-155, 2000.

51. Auvinen P, Tammi R, Parkkinen J, et al: Hyaluronan in peritumoral stroma and malignant cells associates with breast cancer spreading and predicts survival. Am J Pathol 156: 529-536, 2000.

52. Pirinen R, Tammi R, Tammi M, et al: Prognostic value of hyaluronan expression in non-small-cell lung cancer: Increased stromal expression indicates unfavorable outcome in patients with adenocarcinoma. Int J Cancer 95: 12-17, 2001.

53. Posey JT, Soloway MS, Ekici S, et al: Evaluation of the prognostic potential of hyaluronic acid and hyaluronidase (HYAL1) for prostate cancer. Cancer Res 63: 2638-2644, 2003.

54. Fieber C, Baumann P, Vallon R, et al: Hyaluronanoligosaccharide-induced transcription of metalloproteases. J Cell Sci 117: 359-367, 2004.

55. Voelcker V, Gebhardt C, Averbeck M, et al: Hyaluronan fragments induce cytokine and metalloprotease upregulation in human melanoma cells in part by signalling via TLR4. Exp Dermatol 17: 100-107, 2008.

56. West DC, Hampson IN, Arnold F and Kumar S: Angiogenesis induced by degradation products of hyaluronic acid. Science 228 : 1324-1326, 1985.

57. Sugahara KN, Murai T, Nishinakamura H, Kawashima H, Saya $\mathrm{H}$ and Miyasaka M: Hyaluronan oligosaccharides induce CD44 cleavage and promote cell migration in CD44-expressing tumor cells. J Biol Chem 278: 32259-32265, 2003.

58. Duterme C, Mertens-Strijthagen J, Tammi M and Flamion B: Two novel functions of hyaluronidase-2 (Hyal2) are formation of the glycocalyx and control of CD44-ERM interactions. J Biol Chem 284: 33495-33508, 2009.
59. Thomas L, Byers HR, Vink J and Stamenkovic I: CD44H regulates tumor cell migration on hyaluronate-coated substrate. J Cell Biol 118: 971-977, 1992.

60. Herrera-Gayol A and Jothy S: Adhesion proteins in the biology of breast cancer: contribution of CD44. Exp Mol Pathol 66: 149-156, 1999.

61. Fujii K, Tanaka Y, Hubscher S, Saito K, Ota T and Eto S: Crosslinking of CD44 on rheumatoid synovial cells up-regulates VCAM-1. J Immunol 162: 2391-2398, 1999.

62. Fujisaki T, Tanaka Y, Fujii K, et al: CD44 stimulation induces integrin-mediated adhesion of colon cancer cell lines to endothelial cells by up-regulation of integrins and c-Met and activation of integrins. Cancer Res 59: 4427-4434, 1999.

63. Noble PW, Lake FR, Henson PM and Riches DW: Hyaluronate activation of CD44 induces insulin-like growth factor-1 expression by a tumor necrosis factor-alpha-dependent mechanism in murine macrophages. J Clin Invest 91: 2368-2377, 1993.

64. Fujii K, Tanaka Y, Hubscher S, Saito K, Ota T and Eto S: Crosslinking of CD44 on rheumatoid synovial cells augment interleukin 6 production. Lab Invest 79: 1439-1446, 1999.

65. McKee CM, Penno MB, Cowman M, et al: Hyaluronan (HA) fragments induce chemokine gene expression in alveolar macrophages. The role of HA size and CD44. J Clin Invest 98: 2403-2413, 1996

66. Webb DS, Shimizu Y, Van Seventer GA, Shaw S and Gerrard TL: LFA-3, CD44, and CD45: physiologic triggers of human monocyte TNF and IL-1 release. Science 249: 1295-1297, 1990.

67. Wittig BM, Johansson B, Zoller M, Schwarzler C and Gunthert U: Abrogation of experimental colitis correlates with increased apoptosis in mice deficient for CD44 variant exon 7 (CD44v7). J Exp Med 191: 2053-2064, 2000.

68. Ayroldi E, Cannarile L, Migliorati G, Bartoli A, Nicoletti I and Riccardi C: CD44 (Pgp-1) inhibits CD3 and dexamethasoneinduced apoptosis. Blood 86: 2672-2678, 1995.

69. Allouche M, Charrad RS, Bettaieb A, Greenland C, Grignon C and Smadja-Joffe F: Ligation of the CD44 adhesion molecule inhibits drug-induced apoptosis in human myeloid leukemia cells. Blood 96: 1187-1190, 2000.

70. Bourguignon LY, Zhu H, Shao L and Chen YW: CD44 interaction with tiam1 promotes Rac1 signaling and hyaluronic acid-mediated breast tumor cell migration. J Biol Chem 275: 1829-1838, 2000.

71. Oliferenko S, Kaverina I, Small JV and Huber LA: Hyaluronic acid (HA) binding to CD44 activates Rac1 and induces lamellipodia outgrowth. J Cell Biol 148: 1159-1164, 2000.

72. Cywes C and Wessels MR: Group A Streptococcus tissue invasion by CD44-mediated cell signalling. Nature 414: 648-652, 2001.

73. Fitzgerald KA, Bowie AG, Skeffington BS and O'Neill LA: Ras, protein kinase $C$ zeta, and I kappa B kinases 1 and 2 are downstream effectors of CD44 during the activation of NF-kappa B by hyaluronic acid fragments in T-24 carcinoma cells. J Immunol 164: 2053-2063, 2000.

74. Orian-Rousseau V and Ponta H: Adhesion proteins meet receptors: a common theme? Adv Cancer Res 101: 63-92, 2008.

75. Orian-Rousseau V, Chen L, Sleeman JP, Herrlich P and Ponta H: CD44 is required for two consecutive steps in HGF/c-Met signaling. Genes Dev 16: 3074-3086, 2002.

76. Yu WH, Woessner JF Jr, McNeish JD and Stamenkovic I: CD44 anchors the assembly of matrilysin/MMP-7 with heparin-binding epidermal growth factor precursor and ErbB4 and regulates female reproductive organ remodeling. Genes Dev 16: 307-323, 2002.

77. Pienimaki JP, Rilla K, Fulop C, et al: Epidermal growth factor activates hyaluronan synthase 2 in epidermal keratinocytes and increases pericellular and intracellular hyaluronan. J Biol Chem 276: 20428-20435, 2001.

78. Saavalainen K, Pasonen-Seppanen S, Dunlop TW, Tammi R, Tammi MI and Carlberg C: The human hyaluronan synthase 2 gene is a primary retinoic acid and epidermal growth factor responding gene. J Biol Chem 280: 14636-14644, 2005.

79. Godar S, Ince TA, Bell GW, et al: Growth-inhibitory and tumorsuppressive functions of $\mathrm{p} 53$ depend on its repression of CD44 expression. Cell 134: 62-73, 2008.

80. Wielenga VJ, Smits R, Korinek V, et al: Expression of CD44 in Apc and Tcf mutant mice implies regulation by the WNT pathway. Am J Pathol 154: 515-523, 1999. 